\title{
Causality, Localizability, and Holomorphically Convex Hulls
}

\author{
V. Ya. Fainberg and M. A. Soloviev \\ Academy of Sciences of the USSR, Theoretical Physics, \\ P.N. Lebedev Physical Institute, Moscow, USSR
}

\begin{abstract}
A generalization of local commutativity to the fields with an exponential momentum-space growth $\sim e^{l|| p||}$ is considered. To study the local properties of such fields we associate to each space-time region $\mathcal{O}$ a topology $\tau(\mathcal{O})$ on the test function space. It is shown that under any choice of the topology the fields of exponential growth are localizable only in space-time regions large in comparison with $l$. This happens because not any domain in the space of several complex variables is a domain of holomorphy. However, by specifying the topology through the use of holomorphically convex domains in $\mathbb{C}^{4}$, one can attach certain meaning to local commutativity for arbitrarily close spacelike separated regions of $\mathbb{R}^{4}$.
\end{abstract}

\section{Introduction}

A little more than 10 years ago Meyman [1] and Jaffe [2] showed that in the momentum representation the exponential growth of vacuum expectation values is physically marked, since beginning from this growth the fields change essentially their local properties. More precisely, there exist two natural boundaries. The first one characterizes the fields whose domain of definition contains test functions with compact support. This boundary was found by Jaffe with the aid of the theory of quasianalytic classes and looks like this

$$
\int_{1}^{\infty} \frac{\ln g(t)}{t^{2}} d t<\infty .
$$

Here $g$ is a function characterizing the growth in $p$-representation. For example, the growth as $\exp \left\{\|p\| /(\ln \|p\|)^{1+\varepsilon}\right\}$ meets this requirement, whereas $\exp \{\|p\| / \ln \|p\|\}$ does not. Since in the test function space different operations are determined, the presence of only one function of compact support leads automatically to a rather large store of such functions. In particular, in the Jaffe spaces the following important property holds. For any pair of bounded space-time regions $\mathcal{O}_{1}, \mathcal{O}_{2}$ with disjoint closures one can find a test function which is equal to zero in $\mathcal{O}_{1}$ and to unity 


$$
\text { in } \mathcal{O}_{2} \quad \varphi(x)=\left\{\begin{array}{lll}
0 & \text { for } & x \in \mathcal{O}_{1} \\
1 & \text { for } & x \in \mathcal{O}_{2} .
\end{array}\right.
$$

This property makes it possible to construct a partition of unity and the local properties of the corresponding fields turn out to be exactly the same as one of Schwartz distributions. The local commutativity condition for such fields, which are called strictly localizable, is formulated in a usual way

$$
\begin{aligned}
{\left[A\left(\varphi_{1}\right), A\left(\varphi_{2}\right)\right]=0 } & \text { if } \operatorname{supp} \varphi_{1} \text { and } \operatorname{supp} \varphi_{2} \\
& \text { are spacelike separated }
\end{aligned}
$$

Meyman has considered [1] a wider class of functionals growing at infinity slower than any linear exponential

$$
g(t)<C_{\varepsilon} e^{\varepsilon|t|} .
$$

The test functions are analytic and the definition (3) loses its sense in this case. However such fields may be considered as hyperfunctions and both the supports of vacuum expectation values and local commutativity for them can be determined also in the spirit of hyperfunction theory [3]. We shall call them hereafter localizable. The relation between Meyman's and Jaffe's approaches is considered in detail in [4-6]. It is shown there that localizability may be treated as a topological generalization of strict localizability. Namely, to each region $\mathcal{O}$ of space-time a topology $\tau(\mathcal{O})$ on the test functions space is associated and condition (3) is replaced by the following requirement

$$
\begin{aligned}
{\left[A\left(\varphi_{v}^{1}\right), A\left(\varphi_{v}^{2}\right)\right] \rightarrow 0 \quad \text { if } \varphi_{v}^{1} } & \rightarrow 0 \text { in } \tau\left(\mathcal{O}_{1}\right), \\
\varphi_{v}^{2} & \rightarrow 0 \text { in } \tau\left(\mathcal{O}_{2}\right),
\end{aligned}
$$

where $\mathbb{R}^{4} \backslash \mathcal{O}_{1}, \mathbb{R}^{4} \backslash \mathcal{O}_{2}$ are spacelike separated. The topologies $\tau(\mathcal{O})$ are defined in accordance with the growth (4) and are the topologies of compact convergence on arbitrarily close complex neighbourhoods of real sets $\mathcal{O}$. For any pair of regions $\mathcal{O}_{1}, \mathcal{O}_{2}$ with disjoint closures one can find (see [4] and hereafter) a sequence of test functions converging to zero in the topology $\tau\left(\mathcal{O}_{1}\right)$ and to unity in $\tau\left(\mathcal{O}_{2}\right)$

$$
\varphi_{v} \rightarrow\left\{\begin{array}{lll}
0 & \text { in } & \tau\left(\mathcal{O}_{1}\right) \\
1 & \text { in } & \tau\left(\mathcal{O}_{2}\right)
\end{array}\right.
$$

This condition will be called "localizability condition".

The present paper is aimed at clarifying how the local properties change under passing to the exponential growth

$$
e^{l|| p||}
$$

with a finite type $l>0$. The configuration-space test functions are analytic in the tube $\{x+i y:\|y\|<l\}$ in this case and the functionals may be given by complex measures concentrated in this tube. We should emphasize that we are interested in space-time description of these objects, therefore the basis for our approach is the mapping $\mathcal{O} \rightarrow \tau(\mathcal{O}), \mathcal{O} \subset \mathbb{R}^{4}$, which differs it essentially from the mathematical papers by Martineau and Kiselman [8] who studied local properties of analytical functionals in the complex space $\mathbb{C}^{n}$. 
The difficulty of the analysis of local properties intrinsic in the growth (7) is that in the choice of topologies $\tau(\mathcal{O})$ there appears a certain arbitrariness. We shall investigate this arbitrariness and show that the fields with such a growth are not localizable in arbitrarily small space-time regions. The deciding role here is played by holomorphically convex hulls of the complex domains determining the topologies $\tau(\mathcal{O})$. In the one-dimensional case, when every domain is holomorphically convex, the topologies can be chosen so as to achieve a formal coincidence with the local properties of Meyman localizable functionals. We shall describe this choice of the topologies in Section 2 and illustrate by this example a difference between multidimensional and one-dimensional cases. In Section 3 we show that holomorphically convex hulls cannot contain points at distance more than $l$ from the initial domain. Therefore to coarse scales of space-time the local properties of the fields of exponential growth are clearly enough defined. Section 4 considers topologies determined by holomorphically convex domains in $\mathbb{C}^{4}$. Such a choice of topology is of interest due to the fact that condition (6) holds in this case and the corresponding generalization (5) seems to be most near to microcausality. However, under this choice of topology the functionals of growth (7) also are only quasilocalizable since they cannot be decomposed into functionals localized in arbitrarily small space-time regions.

In papers $[6,7]$ we apply the present results to Wightman field theory. In paper [7] we consider in detail a mechanism of violation of microcausality due to the uncertainty in local properties. In [6] it is shown that the condition (5) leads to the symmetry of Wightman functions in their domain of analyticity independently of a freedom of the choice of topology. The existence of a non-empty domain of analyticity for the Wightman functions with the exponential growth (7) has been established in [9]. In the same paper and in [10-12] it has been shown that the symmetry guarantees for the theory of exponential growth certain properties important for physical interpretation. These include the connection between spin and statistics, PCT-theorem, the existence of unitary $S$ matrix, the polynomial boundedness of the elastic scattering amplitude.

\section{The Role of Holomorphically Convex Hulls}

Let $\mathfrak{A}_{l}$ be the space of functions analytic in the tube

$$
\left\{z \in \mathbb{C}^{n}:\|\operatorname{Re} z\|<\infty,\|\operatorname{Im} z\|<l\right\},
$$

where $\|\cdot\|$ is the Euclidean norm. Let us provide it with a topological structure in the following way. To each open set $\mathcal{O} \subset \mathbb{R}^{n}$ we associate the complex domain

$$
\mathcal{O}_{l}=\left\{z \in \mathbb{C}^{n}: \operatorname{Re} z \in \mathcal{O},\|\operatorname{Im} z\|<l\right\}
$$

and define $\tau(\mathcal{O})$ to be the topology of uniform convergence on compact subsets of $\mathcal{O}_{l}{ }^{1}$

$$
\varphi_{v} \rightarrow 0 \text { in } \tau(\mathcal{O}) \Leftrightarrow \sup _{K}\left|\varphi_{v}(z)\right| \rightarrow 0 \text { on every compact } K \subset \mathcal{O}_{l} .
$$

To simplify the exposition we do not impose any restrictions on the behaviour of test functions at infinity. For the definition of topologies $\tau(\mathcal{O})$ with the account taken of fall-off properties see $[4,6]$. For bounded $\mathcal{O}$ it is the same 
A functional $f \in \mathfrak{U}_{l}^{\prime}$ will be regarded as concentrated on the closed set $M \subset \mathbb{R}^{n}$ if it is continuous in each topology $\tau(\mathcal{O})$ where $\mathcal{O} \supset M$. In particular, according to this definition a functional is concentrated in $\mathbb{R}^{4}$ on the projection of support of any complex measure that represents this functional. Now fix two disjount regions $\mathcal{O}_{1}$, $\mathcal{O}_{2} \subset \mathbb{R}^{n}$ and consider the region $\Omega=\mathcal{O}_{1 l} \cup \mathcal{O}_{2 l}$. The complement of this region is connected in $\mathbb{C}^{n}$. In one-dimensional case by virtue of Runge theorem [14] this property makes it possible to approximate any function which is analytic in $\Omega$ by entire functions uniformly on compacts in $\Omega$. In particular, by approximating the function which is equal to zero in $\mathcal{O}_{1 l}$ and equal to unity in $\mathcal{O}_{2 l}$, we make sure that for $n=1$ the localizability condition (6) holds. Moreover one can show that among the closed sets on which a fixed functional is concentrated there exists the smallest one, i.e. the topologies $\tau(\mathcal{O})$ allow us to define self-consistently the support of a functional.

In the multidimensional case the situation changes radically. This may be easily seen on the example of the functional $f=\delta\left(x_{1}^{2}+x_{2}^{2}-1\right)$. An analogous example with pseudo-Euclidean square $x_{1}^{2}-x_{2}^{2}$ is discussed in [7]. Changing to the angular variable one can write the value of the functional $f$ on a test function $\varphi\left(x_{1}, x_{2}\right)$ in the form

$$
(f, \varphi)=\frac{1}{2} \int_{0}^{2 \pi} \varphi(\cos \theta, \sin \theta) d \theta
$$

The integration contour in the plane $\zeta=\theta+i \eta$ can be deformed in such a way that the corresponding contour in the two-dimensional space $\left(z_{1}, z_{2}\right)$ remains within the domain of analyticity of $\varphi$. In particular, distorting to the contour composed of the pieces

$$
\begin{aligned}
& C_{1}=\left(\zeta: \theta=0,0 \leqq \eta<\eta_{0}\right\} \\
& C_{2}=\left\{\zeta: 0 \leqq \theta \leqq 2 \pi, \eta=\eta_{0}\right\} \quad\left(\operatorname{sh} \eta_{0}<l\right) \\
& C_{3}=\left\{\zeta: \theta=2 \pi, 0 \leqq \eta<\eta_{0}\right\}
\end{aligned}
$$

and considering that the integrals over $C_{1}$ and $C_{3}$ compensate each other, we obtain the representation

$$
(f, \varphi)=\frac{1}{2} \int_{0}^{2 \pi} \varphi\left(\operatorname{ch} \eta_{0} \cos \theta-i \operatorname{sh} \eta_{0} \sin \theta, \operatorname{ch} \eta_{0} \sin \theta+i \operatorname{sh} \eta_{0} \cos \theta\right) d \theta .
$$

The corresponding contour in $\mathbb{C}^{2}$ is contained in the compact

$$
K=\{x+i y:\|x\|=\varrho,\|y\| \leqq \lambda\},
$$

where the abbreviations $\varrho=\operatorname{ch} \eta_{0}, \lambda=\operatorname{sh} \eta_{0}$ are used. Its projection onto the real space is a circle of radius $\varrho>1$. Denote the region $x_{1}^{2}+x_{2}^{2}>\varrho^{2}-\varepsilon$ by $\mathcal{O}_{1}$ and the region $x_{1}^{2}+x_{2}^{2}<1+\varepsilon$ by $\mathcal{O}_{2}$. If $\varepsilon$ is small enough these regions are separated by a finite distance, while the sequence $\varphi_{v}$ with the property (6) does not exist for them, since according to the represenation (9) the value of the functional $f$ on such a sequence should tend to $\pi$ and according to the representation (10) to zero. This means that in the two-dimensional case the support cannot be defined with the aid of topologies (8). Indeed, the functional $f$ can be equally considered as concentrated 
on any circle of radius $\varrho$ lying in the interval

$$
1 \leqq \varrho<\sqrt{1+l^{2}} .
$$

The essence of the matter is that the compact (11) is not holomorphically convex in $\mathbb{C}^{2}$. In other words there exist such points $z \notin K$, that for any entire function $\varphi$

$$
|\varphi(z)| \leqq \sup _{\boldsymbol{K}}|\varphi| .
$$

Since entire functions are dense in $\mathfrak{A}_{l}$, this inequality holds also for any test function. Therefore uniform convergence $\varphi_{v} \rightarrow 0$ on $K$ implies convergence to zero in a greater region $\hat{K}$ which is the union of $K$ and all the points for which (13) holds. The set $\hat{K}$ is called the holomorphically convex hull of $K$. We shall now calculate the hull for the compact (11) and show that in accordance with (12) it contains the real set $\varrho^{2}-\lambda^{2} \leqq\|x\|^{2} \leqq \varrho^{2}$. Therefore convergence to zero in the topology $\tau\left(\mathcal{O}_{1}\right)$ in the above example implies convergence to zero on the initial circle $x_{1}^{2}+x_{2}^{2}=1$ though it does not belong to $\mathcal{O}_{1}$. The situation with the $n$-dimensional case is analogous, for any non-convex region $\mathcal{O} \subset \mathbb{R}^{n}$. This is easily seen from the known Levi and Bochner theorems $[13,14]$. The exact formulation of the Levi theorem is not needed, it is just of importance that it allows us to express the holomorphic convexity of a region as a local property of its boundary. The region $\mathcal{O}_{l}$ has a part of the boundary common with the tube $\mathcal{O}_{\infty}=\left\{z \in \mathbb{C}^{n}: \operatorname{Re} z \in \mathcal{O}\right\}$. Therefore these two regions are simultaneously either holomorphically convex or not. According to the Bochner theorem the holomorphic convexity of the tube $\mathcal{O}_{\infty}$ is equivalent to the linear convexity of its base which is the space-time region $\mathcal{O}$. In particular, one can state that convergence to zero in the topology associated according to (8) with the cone $\left\{x \in \mathbb{R}^{4}: x_{0}^{2}>\|\boldsymbol{x}\|^{2}\right\}$ implies convergence to zero in a region containing spacelike points.

Theorem 1. The holomorphically convex hull of the compact $K$ defined by (11) consists of the points $z \in \mathbb{C}^{2}$ satisfying the inequalities

$$
\|x\|^{2}-\|y\|^{2} \geqq \varrho^{2}-\lambda^{2}, \quad\|x\| \leqq \varrho .
$$

Proof. If a point $z^{0}$ does not satisfy inequalities (14), it is easily to find an entire function bounded on $K$ by unity, which is greater than 1 in absolute value at $z^{0}$. In fact, if $\left\|x^{0}\right\|>\varrho$, the corresponding function is

$$
\exp \left\{a_{1} z_{1}+a_{2} z_{2}-\varrho^{2}\right\} \quad \text { with } \quad a_{1}=\frac{x_{1}^{0}}{\left\|x^{0}\right\|} \varrho, \quad a_{2}=\frac{x_{2}^{0}}{\left\|x^{0}\right\|} \varrho .
$$

If $\left\|x^{0}\right\|^{2}-\left\|y^{0}\right\|^{2}<\varrho^{2}-\lambda^{2}$, such a function can be taken to be

$$
\exp \left\{\varrho^{2}-\lambda^{2}-z_{1}^{2}-z_{2}^{2}\right\} \text {. }
$$

Thus, the hull $\hat{K}$ is contained in the set (14). Prove now the converse inclusion. The arguments are in the main the same as ones used in [14] in proving Bochner theorem.

Let us fix a number $r \neq 0, \varrho^{2}-\lambda^{2} \leqq r \leqq \varrho^{2}$ and consider the surface

$$
S_{r}=\left\{z \in \mathbb{C}^{2}: z_{1}^{2}+z_{2}^{2}=r,\|x\| \leqq \varrho\right\} .
$$


Let us show that $S_{r} \subset \hat{K}$. In $S_{r}$ we have

$$
\|y\|^{2}=\|x\|^{2}-r \leqq \lambda^{2} .
$$

From this it follows that the boundary of the surface $S_{r}$ belongs to $K$. Let $\varphi(z)$ be any entire function and $C$ the maximum of $|\varphi|$ in compact $S_{r}$. We must make sure that maximum is attained on the boundary of $S_{r}$. Designate the interior of this set by $S_{r}$. If the maximum is attained in an interior point, the set $\left\{z \in \dot{S}_{r}:|\varphi(z)|=C\right\}$ is not empty. This set is apparently closed in $\stackrel{\circ}{S}_{r}$. On the other hand, it is open in $\stackrel{\circ}{S}_{r}$. In fact, let $z^{0}$ belong to this set. At the point $z^{0}$ either $\frac{\partial}{\partial z_{1}}\left[z_{1}^{2}+z_{2}^{2}-r\right] \neq 0$, or $\frac{\partial}{\partial z_{2}}\left[z_{1}^{2}+z_{2}^{2}\right.$ $-r] \neq 0$, since these derivatives can vanish simultaneously only at the origin which does not belong to $S_{r}$ due to the condition $r \neq 0$. We assume for definiteness that a nonzero derivative is $\frac{\partial}{\partial z_{1}}$. Then by virtue of the implicit function theorem the point $z^{0}$ has such a neighbourhood $V$ in $\stackrel{\circ}{S}_{r}$, that the conditions $z \in V, z_{1}^{2}+z_{2}^{2}=r$ are equivalent to the conditions $z_{1} \in W, z_{2}=f\left(z_{1}\right)$, where $W$ is a neighbourhood of $z_{1}^{0}$ in $\mathbb{C}^{1}$ and $f$ is analytic in $W$. By the maximum principle the function $\psi\left(z_{1}\right)=\varphi\left(z_{1}, f\left(z_{1}\right)\right)$ is constant in $W$ and therefore, the initial $\varphi$ is constant in $V$, i.e. $z^{0}$ is contained in $\left\{z \in \stackrel{\circ}{S}_{r}:|\varphi(z)|=C\right\}$ with this neighbourhood. Thus, the function $\varphi$ is constant in the corresponding component of $S_{r}$ and therefore assumes the same value on its boundary. If $r>0$ there exists only one such component and if $r<0$ there are two, but their amount is not essential for us. It is important only that their boundary are contained in $K$ and thus the inclusion $S_{r} \subset K$ is proven.

By the same arguments the set

$$
S_{r t}=\left\{z \in \mathbb{C}^{2}: z_{1}^{2}+z_{2}^{2}=r+i t,\|x\| \leqq \varrho\right\}
$$

belongs to the hull $\hat{K}$ for any real $t$. The union of these two-dimensional surfaces over all $t$ is the three-dimensional surface

$$
\left\{z \in \mathbb{C}^{2}:\|x\|^{2}-\|y\|^{2}=r,\|x\| \leqq \varrho\right\} .
$$

In turn, the union of these hypersurfaces over $r$ lying in the interval $\varrho^{2}-\lambda^{2} \leqq \mathrm{r} \leqq \varrho^{2}$ is just the set (14). For $\lambda \geqq \varrho$ an exceptional case $r=0$ is possible to which our arguments are inapplicable. However, the set $\hat{K}$ is closed by the very definition. Therefore, the inclusion $S_{r} \subset \hat{K}$ for $r$ close to zero implies the inclusion $S_{0} \subset \hat{K}$. This completes the proof of Theorem 1 .

The same inequalities (14) define the hull of the compact $\{x+i y:\|x\|=\varrho,\|y\|$ $\leqq \lambda\}$ also in the $n$-dimensional case. To prove this it is sufficient to note that this compact is invariant under rotations of $x$ and $y$ and by a simultaneous rotation any point $z \in \mathbb{C}^{n}$ can be carried into the subspace $\left(z_{1}, z_{2}\right)$.

Corollary. Let $\mathcal{O}$ be the complement of a ball of radius $r$ in $\mathbb{R}^{n}$ and let to this region is associated the topology $\tau(\mathcal{O})$ on the space $\mathfrak{A}_{l}$ according to the formula (8). If $l>r$, the convergence $\varphi_{v} \rightarrow 0$ in $\tau(\mathcal{O})$ implies convergence to zero of the numerical sequence $\varphi_{v}(x)$ everywhere inside the ball.

If we consider a field defined on the test function space $\mathfrak{A}_{l}$, this means that it cannot be localized with the aid of topologies (8) in space-time regions small in comparison with $l$. 


\section{Local Properties on Large Space-Time Scales}

Theorem 2. Let $\mathcal{O}$ be a region in $\mathbb{R}^{n}$ and $\mathcal{O}_{l}=\left\{z \in \mathbb{C}^{n}: \operatorname{Re} z \in \mathcal{O},\|\operatorname{Im} z\|<l\right\}$. The holomorphically convex hull of the set $\mathcal{O}_{l}$ in $\mathbb{C}^{n}$ is contained in the closed complex $l$ neighbourhood of the set $\mathcal{O}$.

Proof. Fix a point $\xi \in \mathbb{R}^{n}$ at distance more than $l$ from $\mathcal{O}$ and consider the domain

$$
\Omega_{\xi}=\left\{z \in \mathbb{C}^{n}:\|\xi-\operatorname{Re} z\|>\|\operatorname{Im} z\|,\|\operatorname{Im} z\|<l\right\} .
$$

Evidently, this domain contains $\mathcal{O}_{l}$ and by virtue of what has been said above it is holomorphically convex. The intersection of all such domains is also holomorphically convex and hence it contains the hull $\hat{\mathcal{O}}_{l}$. On the other hand, it is contained in the closed complex $l$-neighbourhood of the set $\mathcal{O}$.

Corollary. Let $\mathcal{O}_{1}, \mathcal{O}_{2}$ be two arbitrary space-time regions separated by a distance more than $2 l$. One can always find for them in $\mathfrak{H}_{l}$ a sequence $\varphi_{v}$ with the property (6).

Proof. The interiors of holomorphically convex hulls $\hat{\mathcal{O}}_{1 l}$ and $\hat{\mathcal{O}}_{2 l}$ are disjoint Runge domains and one can apply to them the approximation theorem.

The proven theorem implies that a field defined on the test function space $\mathfrak{A}_{l}$ is localizable on space-time scales large compared to $l$. This gives us some grounds to think that for sufficiently small $l$ the condition (5) ensures macrocausality. In the limiting case $l \rightarrow 0$ the hull shrinks to the very set. Thanks to this the fields with exponential growth of zero type (4) are localizable in arbitrarily small space-time regions. In the other limiting case, $l \rightarrow \infty$, the hull turns into the convex hull and, in particular, if the region $\mathcal{O}$ is the complement of a bounded set, it covers the whole of the complex space.

\section{Another Choice of Topology}

The question arises whether it is possible to localize the functionals defined on $\mathfrak{A}_{l}$ to a high accuracy by replacing in the definition of topology (8) the domains $\mathcal{O}_{l}$ by holomorphically convex domains $\mathcal{O}_{l}^{\prime}$.

For example, to each region $\mathcal{O} \subset \mathbb{R}^{n}$ we can associate the following set

$$
\mathcal{O}_{l}^{\prime}=\bigcap_{\xi \notin \mathcal{O}} \Omega_{\xi}
$$

where $\Omega_{\xi}$ is defined by (15). As an intersection of holomorphically convex domains $\mathcal{O}_{l}^{\prime}$ is also holomorphically convex. The corresponding topologies will be designated by $\hat{\tau}(\mathcal{O})$. Condition (6) is likely to hold now for any regions with disjoint closures since the holomorphically convex hull of the set $\mathcal{O}_{l}^{\prime}$ in $\mathbb{C}^{n}$ coincides with the closure of this set. It is not hard to construct a sequence (6) explicitly

$$
\varphi_{v}(x)=\left(\frac{v}{\sqrt{\pi}}\right)^{n} \int_{\mathscr{O}_{2}} e^{-v^{2}\|x-\xi\|^{2}} d^{n} \xi .
$$

Nevertheless the local properties still differ significantly from the localizable case. To make sure of this we shall use the criterion found in [5] which characterizes the 
local properties more completely than conditions (2), (6). In the strictly localizable case it looks as follows

$$
\mathscr{L}\left(\mathcal{O}_{1} \cup \mathcal{O}_{2}\right)=\mathscr{L}\left(\mathcal{O}_{1}\right)+\mathscr{L}\left(\mathcal{O}_{2}\right) .
$$

Here $\mathcal{O}_{1}, \mathcal{O}_{2}$ are any two open sets in $\mathbb{R}^{n}$ and $\mathscr{L}(\mathcal{O})$ designates the subspace of the test functions localized in $\mathcal{O}$. This condition is the simplest form of the partition of unity and extends condition (2) to the case of regions with non-empty intersection. In conjunction with the dual formula

$$
\mathscr{L}\left(\mathcal{O}_{1} \cap \mathcal{O}_{2}\right)=\mathscr{L}\left(\mathcal{O}_{1}\right) \cap \mathscr{L}\left(\mathcal{O}_{2}\right)
$$

which holds automatically, it implies that the mapping $\mathcal{O} \rightarrow \mathscr{L}(\mathcal{O})$ is a morphism in the category of lattices. Formulae (16); (17) lead to analogous formulae for the functionals. Indeed, let us designate by $\mathscr{L}^{\prime}(M)$ the set of functionals concentrated on the closed set $M \subset \mathbb{R}^{n}$. It is composed by the functionals vanishing on test functions localized outside $M$. Therefore from (16) it follows

$$
\mathscr{L}^{\prime}\left(M_{1} \cap M_{2}\right)=\mathscr{L}^{\prime}\left(M_{1}\right) \cap \mathscr{L}^{\prime}\left(M_{2}\right),
$$

where $M_{i}$ is the complement of $\mathcal{O}_{i}$. Using the Hahn-Banach theorem it is easy to shown that these formulae are actually equivalent to the accuracy of the closures of the subspaces involved. Similarly, to formula (17) corresponds the equality

$$
\mathscr{L}^{\prime}\left(M_{1} \cup M_{2}\right)=\mathscr{L}^{\prime}\left(M_{1}\right)+\mathscr{L}^{\prime}\left(M_{2}\right) .
$$

In the localizable case (4) conditions (16), (17) are no longer meaningful, since all the subspaces $\mathscr{L}(\mathcal{O})$ are trivial. One can, however, find their topological generalizations. The set of locally convex topologies on a test-function space also forms a lattice i.e. a partially ordered set any two of whose elements have a greatest lower bound and a least upper bound. These bounds being desginated by $\wedge$ and $\vee$, the formulae corresponding to conditions (16), (17) in the localizable case take the form

$$
\begin{aligned}
& \tau\left(\mathcal{O}_{1} \cap \mathcal{O}_{2}\right)=\tau\left(\mathcal{O}_{1}\right) \wedge \tau\left(\mathcal{O}_{2}\right), \\
& \tau\left(\mathcal{O}_{1} \cup \mathcal{O}_{2}\right)=\tau\left(\mathcal{O}_{1}\right) \vee \tau\left(\mathcal{O}_{2}\right) .
\end{aligned}
$$

These formulae lead again to relations (18), (19) where the role of $\mathscr{L}^{\prime}(M)$ will be played by the set of functionals continuous in the topology $\tau(M)$ (see $[5,6]$ ).

Let us turn back to the functionals defined on the space $\mathfrak{A}_{l}$. Under the first choice of topology discussed in Section 2 condition (21) holds but (20) is violated. Using holomorphically convex domains we ensure (20) but it is easily seen that condition (21) and therefore (19) are inevitally violated. We show this on the example of the functional $f=\delta\left(z-z_{0}\right)$, where $\operatorname{Re} z_{0}=0,\left\|\operatorname{Im} z_{0}\right\|^{2}=l^{2}-\varepsilon^{2}$. Choose for $\mathcal{O}_{1}$ the half-space $\left\{x \in \mathbb{R}^{n}: x_{1}<l-2 \varepsilon\right\}$, and for $\mathcal{O}_{2}$ the half-space $\left\{x \in \mathbb{R}^{n}: x_{1}>\right.$ $-(l-2 \varepsilon)\}$. The union of $\mathcal{O}_{1}$ and $\mathcal{O}_{2}$ covers the whole of $\mathbb{R}^{n}$ but it is impossible to represent $f$ as $f_{1}+f_{2}$, where $f_{1}$ is continuous in $\hat{\tau}\left(\mathcal{O}_{1}\right)$ and $f_{2}$ in $\hat{\tau}\left(\mathcal{O}_{2}\right)$, since the sequence of test functions

$$
\varphi_{v}(z)=\exp \left\{v\left(\varepsilon^{2}-l^{2}-\sum_{i=1}^{n} z_{i}^{2}\right)\right\}
$$


on which the functional $f$ is equal to unity converges to zero both in $\hat{\tau}\left(\mathcal{O}_{1}\right)$ and in $\hat{\tau}\left(\mathcal{O}_{2}\right)$. In fact, the points of the tube $\|y\|<l$ for which $\operatorname{Re}\left(\varepsilon^{2}-l^{2}-\sum_{i=1}^{n} z_{i}^{2}\right) \geqq 0$ lie in the region $\|y\|^{2} \geqq l^{2}-\varepsilon^{2},\|x\| \leqq \varepsilon$. In particular, since $\varepsilon<l$, they satisfy the inequality $\|y\| \geqq\|x\|+(l-2 \varepsilon)$. Therefore, none of these points is contained in the region $\Omega_{\xi}$, where $\xi_{1}= \pm(l-2 \varepsilon), \xi_{2}=\ldots=\xi_{n}=0$. On the other hand, $\mathcal{O}_{1 l}^{\prime} \subset \Omega_{\xi_{+}}, \mathcal{O}_{2 l}^{\prime} \subset \Omega_{\xi_{-}}$, which proves our assertion.

We see that under this choice of topology functionals defined on $\mathfrak{A}_{l}$ cannot be decomposed into the sum of functionals localized in small space-time regions. It should be emphasized that the region $\mathcal{O}_{2}^{\prime}$ is smaller than $\mathcal{O}_{2}$ and the requirement of continuity in the topology $\hat{\tau}(\mathcal{O})$ is stronger than that in the topology $\tau(\mathcal{O})$.

\section{Conclusion}

Thus, the fields of exponential growth (7) are localizable only in space-time regions large in comparison with $l$ and the mechanism of localizability violation is connected with envelopes of holomorphy. This mechanism must be taken into account in investigating the relation between the topological generalization of local commutativity (5) and physical causality. Our results show that the problem of causal influence of space-time regions can be correctly set for the fields of exponential growth if the regions are large and separated by a distance greater than $l$. The corresponding generalization of local commutativity (5) provides for the validity of causality, generally speaking, only on space-time scales large compared to $l$. Violations of causality at small distances can come from the fact that the envelope of holomorphy of the complex domain associated with the light cone contains spacelike points. To eliminate such violations one has to use a holomorphically convex domain whose trace in the real space coincides with the light cone. It seems to be the only possible way to ensure causality in the essentially nonlocalizable case $l \rightarrow \infty$. However, one should investigate whether this choice of topology is compatible with other axioms of quantum field theory.

The causality problem in nonlocal field theories was also considered in [15-18]. Efimov and Alebastrov $[15,16]$ studied a theory with an exponentially increasing form-factor in the propagator and drew a conclusion that the theory is microcausal for a specific choice of form-factors, viz., for those form-factors which increase when $p^{2} \rightarrow+\infty$ and fall-off when $p^{2} \rightarrow-\infty\left(p^{2}=p_{0}^{2}-p^{2}\right)$. Microcausality has been defined by them in the same spirit as our condition (5), but the convergence of test functions in space-time regions is understood in a different sense. Namely, to each space-time region $\mathcal{O}$ they associate a sequence (called by them the "projecting" one) with the following properties: it converges on the real space to the characteristic function of the region $\mathcal{O}$ and to zero everywhere in the tube $\left\{z \in \mathbb{C}^{4}: \operatorname{Re} z \notin \mathcal{O}\right\}$ [cf. with (6), (20)]. Unfortunately such a generalization of the partition of unity method turns out to be unsuccessful because these two requirements are compatible only if the convergence is non-uniform. This immediately follows from Theorem 1 of our paper. By examining the examples given in $[15,16]$ one can verify that in the projecting sequences infinitely growing peaks appear which narrow and move so as to provide convergence to zero at each point $z$ with $\operatorname{Re} z \notin \mathcal{O}$. This important fact has 
not been taken into account in $[15,16]$, quite the reverse - the property of uniform convergence is assumed and essentially exploited in the proof of microcausality proposed there, which makes it erroneous. In [7] we have studied the form-factors $[15,16]$ by the method presented here and have shown that they cannot be microcausal. We think however that the field theory $[15,16]$ is macrocausal for small $l$ and physically relevant.

In $[17,18]$, topological generalizations of local commutativity have been suggested for those fields that grow in momentum space like $\exp \left\{\|p\|^{\alpha}\right\}$ with $\alpha>1$. The corresponding configuration-space test functions are entire of order $\alpha /(\alpha-1)$ and cannot decrease at $\|x\| \rightarrow \infty$ more rapidly than $\exp \left\{-\|x\|^{\alpha /(\alpha-1)}\right\}$. In [6] the topological structure used in $[17,18]$ is considered from the viewpoint of criterion (6), (20) and it is shown that all the topologies corresponding to different bounded regions are equivalent. Only the topologies corresponding to different cones differ from one another. In other words that topological structure distinguishes only between directions but not between points in space-time. The causality problem in such a theory requires a more detailed consideration. We would like to emphasize again that that choice of topology is not the only possible one.

It is of interest that the Haag-Ruelle scattering theory can be completely extended to nonlocalizable fields with arbitrarily fast growth $[11,19,20]$ and turns out to be nonsensitive to the manner of spacelike falling the field commutator. However the exponential growth (7) plays a particular role here too. Namely, only for finite $l$ the Wightman functions have a non-empty domain of analyticity. This domain contains real points (Jost points) [9]. By using delta-like sequences of test functions instead of functions of compact support and applying the CauchyPoincare theorem, we have proved [6] that generalized local commutativity (5) implies the symmetry of the Wightman functions under permutations of the arguments independently of an arbitrariness of the choice of topology. The localizable case $(l \rightarrow 0)$ was also treated by Lücke [21] in a similar way. The symmetry leads in the usual fashion to the correct connection between spin and statistics and PCT-invariance. When $l \rightarrow \infty$, the domain of analyticity vanishes and these important properties of asymptotic fields cannot be derived without additional assumptions.

\section{References}

1. Meyman, N.N.: ZhETF 47, 1966 (1964) [English transl. : Sov. Phys.-JETP 20, 1320 (1965)]; ITEP Preprint No. 252 (1964)

2. Jaffe, A.M.: Phys. Rev. Lett. 17, 661 (1966); Phys. Rev. 158, 1454 (1967)

3. Nagamachi, S., Mugibayashi,N.: Commun. math. Phys. 46, 119-134 (1976)

4. Soloviev, M. A.: Theor. Math. Phys. (USSR) 7, 183-191 (1971)

5. Soloviev, M. A.: Theor. Math. Phys. 20, 299-301 (1974)

[English transl.: Sov. J. Theor. Math. Phys., p. 835 (June 1975)]

6. Soloviev, M. A., Fainberg, V. Ya. : In :Proceedings of the IVth International Symposium on Nonlocal Field Theories, JINR D2-9788, Dubna, pp. 57-76 (1976); Lebedev Phys. Inst. Preprint No. 95 (1977)

7. Soloviev,M.A., Fainberg,V.Ya.: Kratkie soobshcheniya po fizike, FIAN, No. 1, 17-22 (1977) [English transl.: Lebedev Inst. Reports (to appear)]

8. Martineau, A.: J. Analyse Math., Jerusalem, 11, 1-164 (1963)

Kiselman,C.: Arkiv Mat. 6, 307 (1965) 
9. Iofa,M.Z., Fainberg,V.Ya.: ZhETF 56, 1644 (1969) [English transl. : Sov. Phys.-JETP 29, 880-886 (1969)]; Theor. Math. Phys. 1, 187-199 (1969)

10. Iofa, M.Z.: Theor. Math. Phys. 3, 197-211 (1970)

11. Iofa,M.Z., Fainberg,V.Ya.: Nuovo Cimento 5A, 273 (1971)

12. Fainberg,V. Ya.: In: Problems of theoretical physics, pp. 120-145. Moscow: Nauka 1972

13. Vladimirov, V.S. : Methods of the theory of functions of several complex variables. Cambridge: MIT Press 1966

14. Hörmander,L.: An introduction to complex analysis in several variables. Toronto-New YorkLondon: Van Nostrand 1966

15. Efimov,G.V.: JINR Preprint P2-6756, Dubna 1972

16. Alebastrov,V.A., Efimov,G.V.: Commun. math. Phys. 38, 11 (1974); JINR Preprint P2-7572, Dubna 1973

17. Constantinescu,F., Taylor,J.G.: J. Math. Phys. 15, 824 (1974)

18. Bümmerstede,J., Lücke,W.: J. Math. Phys. 16, 1203-1209 (1975)

19. Taylor,J.G.: Ann. Phys. (N.Y.) 68, 484 (1971)

20. Bümmerstede,J., Lücke,W.: Commun. math. Phys. 37, 121 (1974)

21. Lücke,W.: J. Math. Phys. 17, 1515-1520 (1976)

Communicated by R. Haag

Received March 11, 1977; in revised form July 20, 1977 
